SHORT REPORT

\title{
Biphasic cardioversion of acute atrial fibrillation in the emergency department
}

\author{
G K Lo, D M Fatovich, A D Haig
}

Emerg Med J 2006;23:51-53. doi: 10.1136/emj.2004.021055

Introduction: There is a trend towards accelerated management of acute atrial fibrillation (AF) in the emergency department (ED). We report our experience with biphasic cardioversion of acute AF.

Methods: This was a prospective, descriptive study at a tertiary hospital ED over a 6 month period. Acute AF was defined as symptoms that had been present for $<48$ hours. Patients who received biphasic cardioversion for acute AF in the ED were enrolled. Data collected included: patient demographics, past medical history, details of biphasic cardioversion, outcome, complications, disposition, and length of stay.

Results: There were 34 attempts at cardioversion in 33 patients. The mean (SD) age was 56 (16) years and 21 patients (64\%) were men. Biphasic cardioversion was successful in 31 attempts (91\%). In 24 attempts (71\%), $100 \mathrm{~J}$ was selected as the initial energy level. This was successful in 21 attempts (88\%). There were three minor complications related to sedation. The mean (SD) length of stay was $5.6(2.8)$ hours in the ED and $15(25)$ hours in the hospital. The three patients who failed to revert were older (mean age 64 years), had underlying cardiovascular disease, and spent longer in hospital (50v12 hours, $p=0.01$ ). Telephone follow up was conducted with 32 patients (97\%) at 3 months. Recurrence of AF occurred in 7 patients (22\%). Most patients $(31,97 \%$ ) were satisfied with the biphasic cardioversion.

Conclusions: Biphasic cardioversion of acute AF is effective. The majority of patients can be managed as outpatients, and there is very high patient satisfaction with this approach. An initial shock energy level of $100 \mathrm{~J}$ is usually effective.

A trial fibrillation (AF) is the most common arrhythmia seen in the emergency department (ED). Traditionally, most patients with acute AF are admitted to exclude a serious cardiac condition, and to monitor for complications. ${ }^{1}$

Several studies $^{1-5}$ demonstrate that acute AF can be managed successfully in the ED with high short term conversion and discharge rates. Reports have shown that biphasic defibrillators are more effective and deliver less energy than monophasic defibrillators. ${ }^{67}$ These studies were performed in a cardiology outpatient setting, and there are very few data describing biphasic cardioversion of acute AF in the ED.

We report the effectiveness and outcome of biphasic cardioversion of acute AF in a tertiary referral ED.

\section{METHODS}

A prospective, descriptive study was conducted at Royal Perth Hospital Emergency Department from 20 January to 20 July 2003. All patients with acute AF who received biphasic cardioversion were enrolled. Acute AF was defined as AF in which symptoms had been present for $<48$ hours.

The patient was managed in a monitored area with cardiac monitoring. Low molecular weight heparin was given to all patients unless they were already anticoagulated. Patients were assessed by the emergency physician for electrical cardioversion. Factors such as underlying medical conditions and contraindications (symptoms for more than 48 hours, a history of chronic AF, thromboembolic complications, or a high risk of thromboembolism) were considered.

The agents used for sedation were propofol or fentanyl and midazolam. The drugs and doses were chosen based on patient characteristics and titrated accordingly. The machine used was the Medtronic Physio-Control Lifepak 12, which delivers a biphasic truncated exponential waveform. Patients were observed in the ED until they were safe for discharge. Patients who were suspected to have acute coronary syndrome were further investigated.

The key outcomes were rate of conversion, energy level used, time from admission to biphasic cardioversion, length of stay in the ED and the hospital, and rate of hospital discharge. Telephone follow up was performed at 3 months after discharge. Data collected were recurrence of AF, incidence of thromboembolic complications, and patient satisfaction. Satisfaction was assessed by asking if the patient would choose biphasic cardioversion for a recurrence.

\section{Statistical analysis}

All the data were recorded and analysed with SPSS (version 11; SPSS Inc, Chicago, IL, USA). Standard descriptives, including mean, median, and standard deviations, were obtained and percentages were given. Statistical comparison was made between the successful and failed cardioversions.

\section{RESULTS}

During the study period, 224 ED attendances were identified with a diagnosis of AF of any duration. The overall results and disposition are summarised in fig 1. There were 34 attempts at biphasic cardioversion for AF in 33 patients (one of the patients presented twice and was treated with biphasic cardioversion on both occasions).

Patient demographics and clinical parameters are presented in table 1. ED treatment summary is presented in table 2 . There were $31(91 \%)$ successful cardioversions out of 34 attempts.

There were three episodes of minor complications related to procedural sedation. One patient developed laryngospasm, which resolved spontaneously, one patient was oversedated, and one developed hypotension, which responded to intravenous fluid. There were no recorded episodes of oxygen desaturation, nor any thromboembolic complications.

Three patients failed to convert after biphasic cardioversion. The patients who failed were more likely to be older and

Abbreviations: $A F$, atrial fibrillation; $E D$, emergency department 


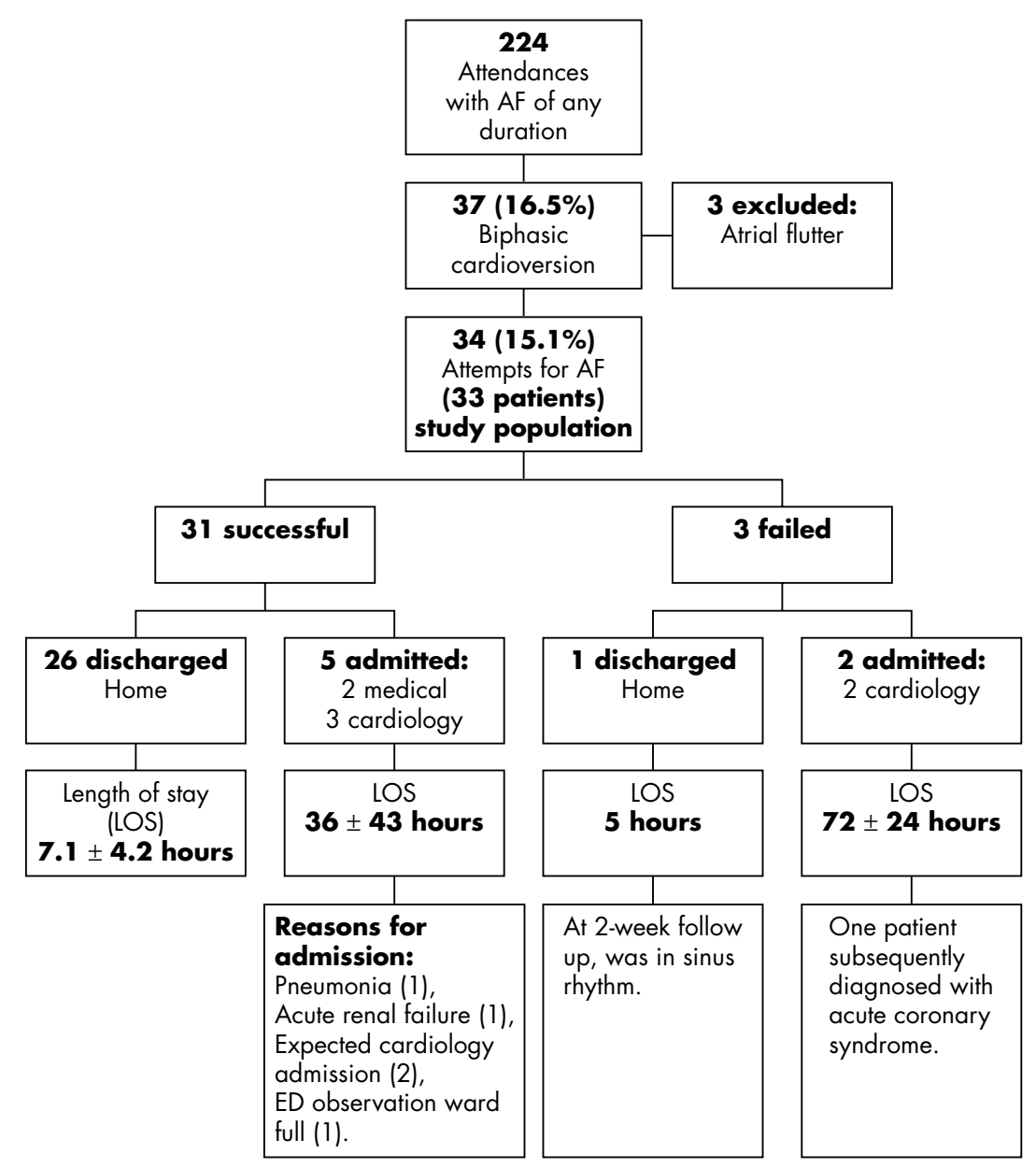

Figure 1 Overall results and disposition.

to have underlying cardiovascular disease compared with the successful group; however, these differences were not statistically significant. The mean length of stay in hospital was longer in the failed group ( $50 v 12$ hours, $\mathrm{p}=0.01$ ).
At 3 months after discharge, 32 patients were contacted. Recurrence of AF had occurred in 7 patients $(22 \%)$, but despite this, 31 patients $(97 \%)$ were satisfied with the biphasic cardioversion procedure.

Table 1 Patient demographics, initial assessment, and management

\begin{tabular}{|c|c|c|c|c|}
\hline Characteristics & $\begin{array}{l}\text { Total } \\
\text { patients } \\
(n=33)^{*}\end{array}$ & $\begin{array}{l}\text { Successful } \\
\text { cardioversion } \\
(n=31)\end{array}$ & $\begin{array}{l}\text { Failed } \\
\text { cardioversion } \\
(n=3)\end{array}$ & p† \\
\hline Mean (SD) age (years) & $56(16)$ & $55(16)$ & $64(9)$ & 0.36 \\
\hline Age range (years) & $31-90$ & $31-90$ & $54-75$ & \\
\hline Men & $21(64 \%)$ & $18(58 \%)$ & 3 & 0.22 \\
\hline \multicolumn{5}{|l|}{ Previous history } \\
\hline Ischaemic heart disease & $8(24 \%)$ & $6(19 \%)$ & 2 & 0.13 \\
\hline Valvular heart disease & $11(33 \%)$ & $10(32 \%)$ & 2 & 0.28 \\
\hline Hypertension & $17(52 \%)$ & $14(45 \%)$ & 3 & 0.11 \\
\hline History of AF & $19(58 \%)$ & $18(58 \%)$ & 2 & 0.64 \\
\hline Heart failure & $2(6 \%)$ & $2(6 \%)$ & 0 & 0.83 \\
\hline $\begin{array}{l}\text { Mean (SD) heart rate on arrival } \\
\text { (beats/min) }\end{array}$ & $136(20)$ & $137(21)$ & $133(12)$ & 0.79 \\
\hline Mean (SD) Systolic bP (mmHg) & $133(27)$ & $133(25)$ & 127 (37) & 0.70 \\
\hline Range of systolic BP (mmHg) & $80-210$ & $84-210$ & $80-170$ & \\
\hline Mean (SD) diastolic BP (mmHg) & $83(17)$ & $83(16)$ & $78(18)$ & 0.66 \\
\hline Concomitant medical problem & $5(15 \%)$ & $4(13 \%)$ & 1 & 0.33 \\
\hline Pneumonia & $1(3 \%)$ & $1(3 \%)$ & 0 & \\
\hline Viral illness & $2(6 \%)$ & $2(6 \%)$ & 0 & \\
\hline Acute renal failure & $1(3 \%)$ & $1(3 \%)$ & 0 & \\
\hline Acute coronary syndrome & $1(3 \%)$ & 0 & 1 & \\
\hline $\begin{array}{l}\text { Use of amiodarone before } \\
\text { biphasic cardioversion }\end{array}$ & $13(38 \%)$ & $10(32 \%)$ & 3 & 0.05 \\
\hline
\end{tabular}




\begin{tabular}{|c|c|c|c|c|}
\hline \multirow[b]{2}{*}{ Characteristics } & \multicolumn{3}{|c|}{ Cardioversions } & \multirow[b]{2}{*}{$\mathbf{p}^{*}$} \\
\hline & $\begin{array}{l}\text { Total } \\
(n=34)\end{array}$ & $\begin{array}{l}\text { Successful } \\
(n=31)\end{array}$ & $\begin{array}{l}\text { Failed } \\
(n=3)\end{array}$ & \\
\hline $\begin{array}{l}\text { Time from attendance to } \\
\text { biphasic cardioversion (hours) }\end{array}$ & $3.1(2.1)$ & $3.1(2.2)$ & $2.5(1.0)$ & 0.65 \\
\hline \multicolumn{5}{|l|}{ Original biphasic energy used (J) } \\
\hline $50 \mathrm{~J}$ & $2(6 \%)$ & $2(6 \%)$ & 0 & \\
\hline 100 & $24(71 \%)$ & $21(68 \%)$ & 3 & \\
\hline 150 & $5(15 \%)$ & $5(16 \%)$ & 0 & \\
\hline 200 & $3(9 \%)$ & $3(10 \%)$ & 0 & \\
\hline \multicolumn{5}{|l|}{ Number of shocks given } \\
\hline 1 & $29(85 \%)$ & $29(94 \%)$ & 0 & \\
\hline 2 & $3(9 \%)$ & $2(6 \%)$ & 1 & \\
\hline 3 & $1(3 \%)$ & 0 & 1 & \\
\hline 5 & $1(3 \%)$ & 0 & 1 & \\
\hline Median no. (range) of shocks given & $1(1-5)$ & $1(1-2)$ & $3(2-5)$ & $0.001 \dagger$ \\
\hline \multicolumn{5}{|l|}{ Complications } \\
\hline Sedation related & $3(9 \%)$ & $3(10 \%)$ & 0 & 0.90 \\
\hline Thromboembolism & 0 & 0 & 0 & \\
\hline \multicolumn{5}{|l|}{ Length of stay (hours) } \\
\hline In ED, mean (SD) & $5.6(2.8)$ & $5.6(2.9)$ & $5.3(1.2)$ & 0.88 \\
\hline In hospital, mean (SD) & $15(25)$ & $12(21)$ & $50(37)$ & $0.01 \dagger$ \\
\hline In hospital, median (range) & & $6(2-120)$ & $48(5-96)$ & 0.15 \\
\hline
\end{tabular}

\section{DISCUSSION}

Our cardioversion success rate of $91 \%$ is high, and consistent with success rates of $69-94 \%$ from recent reports. ${ }^{67}$ Previous retrospective studies ${ }^{1-5}$ are supportive of an accelerated management approach to acute $\mathrm{AF}$, which may lead to a decreased need to admit these patients.

Successful cardioversion was associated with a much shorter length of stay in hospital. Although no formal financial analysis was performed, this practice represents a substantial cost saving for the hospital. Patient satisfaction with the procedure is very high, because cardioversion resolves their problem and hence avoids admission to hospital.

There are no current recommendations on the initial energy level used in biphasic cardioversion. We usually found $100 \mathrm{~J}$ to be effective and recommend that as the starting level.

Recently, there have been several clinical trials demonstrating that rate control is not inferior to rhythm control in AF. ${ }^{8}$ These studies examined patients with significant cardiovascular comorbidities and the patients were older ( $\sim 70$ years), had persistent or permanent $\mathrm{AF}$, and were minimally symptomatic. In our study, the patients were younger ( 56 years), healthier, had acute AF, and were highly symptomatic.

This study has several limitations. The sample size was small and hence did not have sufficient power to detect a rare complication such as thromboembolism (reported rate $0.8 \%) .{ }^{9}$ We did not detail the characteristics of the entire population of patients with $\mathrm{AF}$, because this was beyond the scope of this paper, as we wished to primarily report our experience of biphasic cardioversion of acute AF. There was no formal protocol describing the decision making process, hence there may be a selection bias. This study did not compare biphasic cardioversion with chemical cardioversion and the "wait and watch" approach. Finally, there was a risk of interviewer bias at follow up.

The experience we have gained allows us to develop a management algorithm. This could be tested prospectively in a trial. If that came close to replicating these results, we would have a useful transferable message for others to use.
In summary, we found that biphasic cardioversion of acute AF is effective. The majority of patients can be managed as outpatients, and there is very high patient satisfaction with this approach.

\section{Authors' affiliations}

G K Lo, A D Haig, Royal Perth Hospital, Perth, Australia D M Fatovich, University of Western Australia, Australia

Competing interests: there are no competing interests.

Correspondence to: Dr G Lo, Dept of Emergency Medicine, Royal Perth Hospital, Box X2213 GPO, Perth WA 6001, Australia; godfrey.lo@ health.wa.gov.au

Accepted for publication 12 January 2005

\section{REFERENCES}

1 Michael JA, Stiell IG, Agarwal S, Mandavia DP. Cardioversion of paroxysmal atrial fibrillation in the emergency department. Ann Emerg Med 1999;33:379-87

2 Koenig BO, Ross MA, Jackson RE. An emergency department observation unit protocol for acute-onset atrial fibrillation is feasible. Ann Emerg Med 2002;39:374-81.

3 Kim MH, Morady F, Conlon B, Kronick S, Lowell M, Bruckman D, Armstrong WF, Eagle KA. A prospective, randomized, controlled trial of an emergency department-based atrial fibrillation treatment strategy with lowmolecular-weight heparin. Ann Emerg Med 2002;40:187-92.

4 Domanovits $\mathbf{H}$, Schillinger $M$, Thoennissen J, Nikfardjam M, Janata K, Brunner M, Laggner AN. Termination of recent-onset atrial fibrillation/flutter in the emergency department: a sequential approach with intravenous ibutilide and external electrical cardioversion. Resuscitation 2000;45:181-7.

5 Burton JH, Vinson DR, Drummond K, Strout TD, Thode HC, McInturff JJ. Electrical cardioversion of emergency department patients with atrial fibrillation. Ann Emerg Med 2004;44:20-30.

6 Scholten M, Szili-Torok T, Klootwijk P, Jordaens L. Comparison of monophasic and biphasic shocks for transthoracic cardioversion of atrial fibrillation. Heart 2003:89:1032-4.

7 Khaykin Y, Newman D, Kowalewski M, Korley V, Dorian P. Biphasic versus monophasic cardioversion in shock-resistant atrial fibrillation. J Cardiovasc Electrophysiol 2003;14:868-72.

8 Van Gelder IC, Hagens VE, Bosker HA, Kingma JH, Kamp O, Kingma T, Said SA, Darmanata Jl, Timmermans AJ, Tiissen JG Criins HJ, Rate Control versus Electrical Cardioversion for Persistent Atrial Fibrillation Study Group. A comparison of rate control and rhythm control in patients with recurrent persistent atrial fibrillation. N Engl J Med 2002;347:1834-40.

9 Weigner MJ, Caulfield TA, Danias PG, Silverman DI, Manning WJ. Risk for clinical thromboembolism associated with conversion to sinus rhythm in patients with atrial fibrillation lasting less than 48 hours. Ann Intern Med 1997; 126:615-20. 